\title{
Monitoring drying and wetting of a cement bentonite mixture with Electrical Resistivity Tomography
}

\author{
Guido Musso ${ }^{1, *}$, Antonio Zibisco ${ }^{1}$, Renato Maria Cosentini $^{1}$, Paolo Trischitta $^{1}$, Gabriele Della Vecchia ${ }^{2}$ \\ ${ }^{1}$ Politecnico di Torino, DISEG, Corso Duca degli Abruzzi 24, 10129 Torino, Italy \\ ${ }^{2}$ Politecnico di Milano, DICA, Piazza Leonardo da Vinci 32, 20133 Milano, Italy
}

\begin{abstract}
Cement bentonite slurry cutoff walls are used to encapsulate pollutants within contaminated areas, so avoiding their spreading in the environment. In both temperate and arid climates, at shallow depths, slurry walls are exposed to interaction with the atmosphere and thus to relative humidity values which might induce desaturation and significant shrinkage. This note presents the main results of a study aimed at investigating the impact of drying processes on the integrity and the hydraulic performance of cement bentonite slurry walls. Cement bentonite samples were cured under water for different times ( 1 months, 2 months and 4 months) and then dried naturally by exposing them to the laboratory environment ( $\mathrm{T}=21{ }^{\circ} \mathrm{C}$, relative humidity approximately $38 \%$ ). Once dried, the bottom of the samples was placed in contact with a thin layer of water to induce wetting. The distribution of the electrical conductivity within these samples was evaluated through Electrical Resistivity Tomography measurements, and electrical conductivity maps were converted then into maps of water contents on basis of a phenomenological relationship. The reconstructed water contents compared very well to the measured ones. Drying induced a limited cracking of the samples, which might affect to some extent the hydraulic performance of the barriers.
\end{abstract}

\section{Introduction}

The aim of cement-bentonite (CB) slurry cutoff walls is the containment of dissolved and of non-aqueous liquid pollutants within confined areas. These engineering barriers have been successful in engineering practice because they are very easy to build: trenches are dug around contaminated sites and they are then filled with plastic mixtures of cement and bentonite at a slurry state. Curing occurs within the trench: here the slurry hardens with time, while its strength increases and its permeability decreases [1]. In the mixture, a certain percentage of cement is often replaced by slab or by fly ash, which allows developing a higher strength and a lower permeability [2]. The mechanical behaviour of hardened cement bentonite pastes has been investigated mostly in terms of unconfined compressive strength. However, according to the oedometer compression and triaxial tests shown in [3], the mechanical response of these mixtures once hardened is similar to the one of bonded soils: it is rather stiff below a threshold stress, and highly compressible above it. Collapse of bonds has been claimed to be responsible for the sharp change in the compression behaviour [3].

Although cutoff walls are expected to interact with evolving water tables and atmospheric conditions, a proper understanding of their behaviour in the vadose zone, or after being exposed to drying-wetting cycles, has not been reached yet. Very few studies have dealt with this aspect, with contradictory results. CB samples have been reported to crack when dried through exposure to the laboratory environment but not when exposed to drying wetting cycles while immersed in a box filled with sand $[4,5]$. However, in the latter case also the uniaxial compressive strength was found to be affected by the hydraulic history. No cracks were detected above the water table in a cut-off wall that was examined 8 years after construction [5]. Other evidences [6] do not simply report of cracks developing in samples exposed to the laboratory atmosphere, but also of large cracks found when examining existing barriers whose surface was left uncapped. These cracks were claimed to extend 2 meters below the current phreatic line and they were supposed to be related to the shrinkage caused by drying. Preliminary laboratory tests in [7] support the same conclusions. Interestingly, samples enriched with slab or fly ash seem to show worst performances upon drying than pure cement-bentonite mixtures.

Together with [8], this study aims at a preliminary insight on the drying processes of a cement-bentonite mixture, and particularly to the process of evaporation from cylindrical samples and from a small experimental column. The evolution of water content is monitored by 2D Electrical Resistivity Tomography (ERT), whose use in this context is also assessed.

Corresponding author: guido.musso@polito.it 


\section{Material and methods}

\subsection{Materials}

The cement used in this work is a commercial CEM I $32,5 \mathrm{~N}$, Portland 325, cement. The bentonite is a commercial mixed sodium - calcium bentonite provided by Laviosa Chimica Mineraria (Livorno, Italy). The liquid limit of the bentonite is $\mathrm{w}_{\mathrm{L}}=616 \%$ and the plastic limit is $w_{P}=42 \%$. The specific gravity is $G_{s}=2.95$. The hygroscopic water content of the bentonite powder at laboratory conditions is $\mathrm{w}=14 \%$.

\subsection{Sample preparation}

The bentonite powder was thoroughly mixed with tap water in a mixer to obtain a homogeneous plastic paste with water to solid mass ratio of 18:1 (mass ratio). The paste was covered with an impermeable sheet and left to rest for 24 hours to ensure full hydration of the bentonite; then dry cement powder was added and the resulting suspension was stirred again. A cementbentonite (dry) mass ratio of $4: 1$ was adopted. Ratios used in engineering works are usually in the range $4: 1$ to 6:1. The cement-bentonite mixture was poured into plastic cylindrical moulds of different diameter and height. These cylindrical moulds were laid on the bottom of boxes filled with tap water [8]. Different curing times were imposed (28, 60 and 90 days insofar). The moulds had holes equally spaced on their perimeter, allowing for the insertion and connection of one or more levels of sixteen electrodes centred at half the height of the specimens. The electrodes were thin silver squared foils of approximately $5 \mathrm{~mm}$ sides, welded to silver wires. The electrodes were placed inside the moulds before pouring the mixture. Figure 1 shows a picture of an empty mould with the electrodes in place.

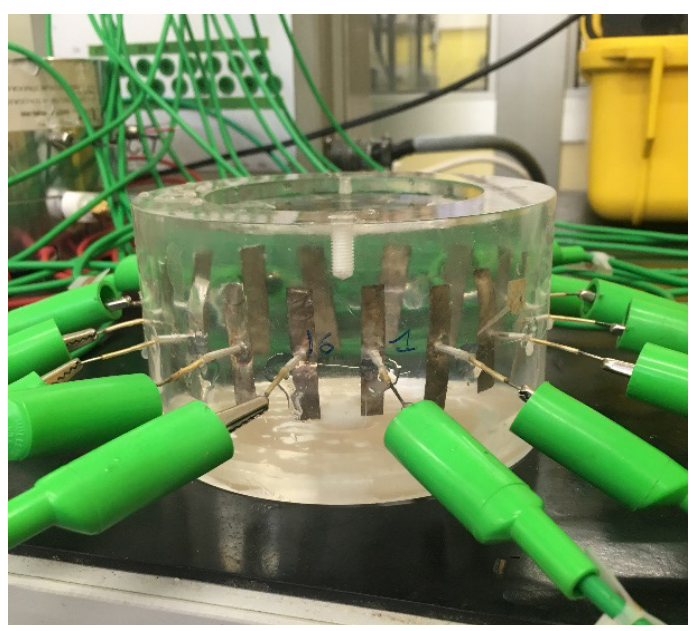

Fig. 1. Picture of plastic mould with electrodes and electrical connections.

\subsection{Electrical Resistivity Tomography}

ERT allows the reconstruction of the electrical conductivity field within an object. This is obtained through inversion of electrical measurements, where two electrodes inject current and other two measure the corresponding drop of electrical potential. A redundant number of measurements is performed, by changing the distance and the position of the injecting and measuring electrodes. The measured potential drops are then compared to a direct FE solution of the electrical problem. An optimization scheme updates the electrical conductivity within the FE model, in order to minimize the difference between experimental measurements and model predictions. The reconstructed electrical conductivity field is then obtained. ERT has been successfully used to detect heterogeneities [9], or to visualize mechanical and transport processes in soil samples [10], also allowing estimating the water retention and hydraulic properties of different soils [11]. It also has been fruitfully used to investigate moisture movement in cement, both undamaged [12] - thus allowing to study the homogeneous behaviour of the material matrix - and cracked [13]. The present study relies on a $2 \mathrm{D}$ reconstruction protocol, where the measurements are taken from 16 electrodes placed on the circumference of the sample (Figure 1). The detailed measurement and reconstruction protocols are given in [9].

\subsection{Electrical conductivity of homogeneous samples}

A preliminary characterization of the electrical behaviour of the cement-bentonite mixture was carried out through one dimensional electrical measurements made on cylindrical specimens of height $\mathrm{H}=80 \mathrm{~mm}$ and section area $S=507 \mathrm{~mm}^{2}$. Two cylindrical electrodes, A and $\mathrm{B}$ in Figure 2, were inserted on the extremities of a cylinder. Other two 'needle' electrodes (M and $\mathrm{N}$ ) were inserted along the side of the specimen at a distance $L=40 \mathrm{~mm}$ one from the other and equally spaced from $\mathrm{A}$ and $\mathrm{B}$. The drop in the electrical potential $\Delta \mathrm{V}$ between $\mathrm{M}$ and $\mathrm{N}$ was measured, while a known DC electrical current of magnitude $I$ was applied by the electrodes A and B. The electrical conductivity of the specimen was then evaluated as:

$$
\chi=\frac{\Delta V}{I} \frac{L}{S}
$$

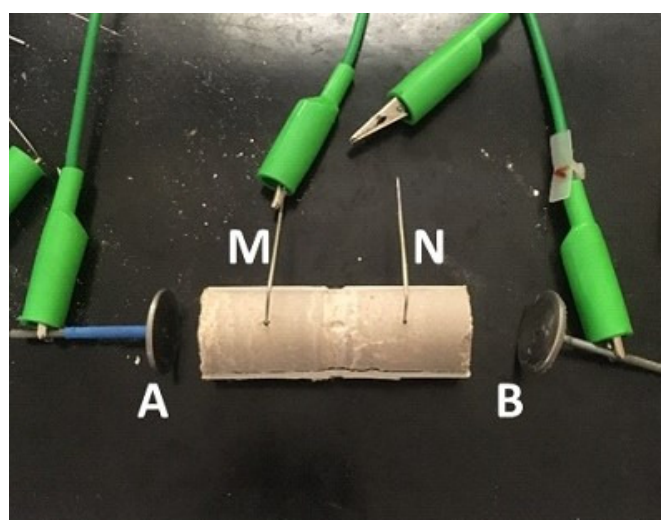

Fig. 2. Electrode arrangement for one dimensional electrical measurements. 


\section{Experimental results}

\subsection{Evolution of the electrical conductivity during curing}

ERT was performed at different curing times, to evaluate the evolution of the electrical conductivity. Samples were taken out of the curing bath and the distribution and average value of the electrical conductivity at different times was determined. A very homogeneous distribution was found at any curing time: the average electrical conductivity initially decreased but remained constant afterwards. The trend in Figure 3 strongly suggests that the fabric of the sample develops gradually during the first 28 days of curing and remains relatively stable afterwards. This interpretation is supported by the results of hydro-mechanical tests performed on samples of different curing age: the hydraulic conductivity, the oedometer compression curves and the strength envelope showed very little dependency on time for $t>28$ days. A very small progressive shift in the compression curves was found, i.e. development of bonded structure even if there are no changes in fabric (Figure 4).

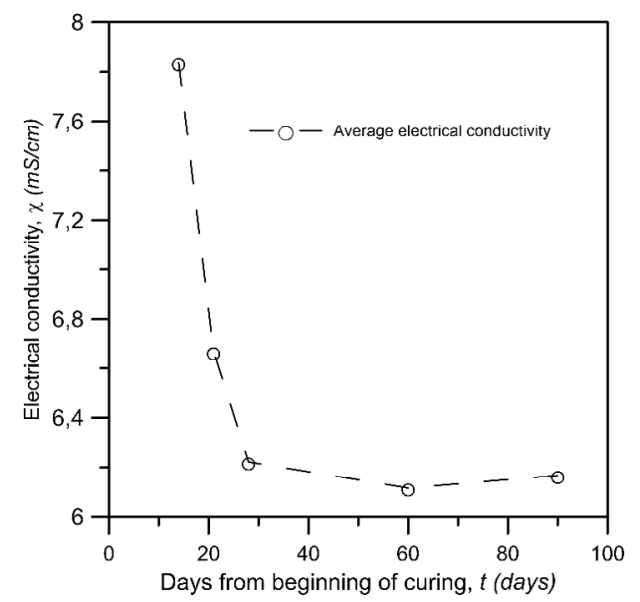

Fig. 3. Evolution of electrical conductivity with time during curing.

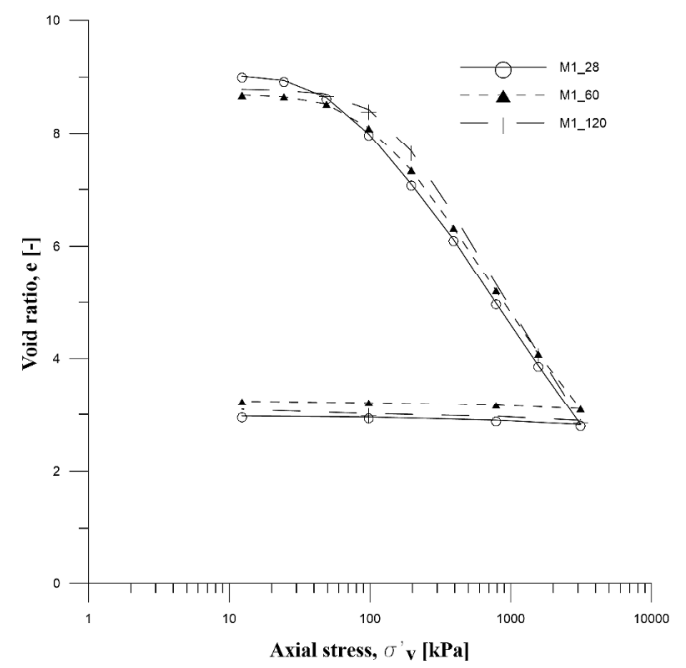

Fig. 4. Oedometer compression curves of samples with 28, 60 and 120 days curing time.

\subsection{Relationship between water content and electrical conductivity}

The electrical conductivity of porous media depends on their fabric and on the electrical properties of the single constituents. In the simplest case, e.g. coarse soils, the contribution of the solid grains is negligible, and the electrical conductivity of the soil is a function of its porosity and degree of saturation, and of the concentration of the charged species dissolved in the pore water. Viceversa, clays have a charged specific surface (whose size depends on clay mineralogy), and thus a relevant surface electrical conductivity. Although various theoretical models exist accounting for the role of fabric and of the different constituents, because of the scarcity of laboratory data concerning the electrical conductivity of unsaturated cement-bentonite mixtures, an interpretation based on an experimental phenomenological law was here preferred.

To this extent, one dimensional measurements were performed adopting the cell of Figure 2. A first measurement was done immediately after removing a sample from the water bath where it was cured (gravimetric water content $w=285 \%$ ). Other measurements were repeated at decreasing water contents, which were imposed by air drying. The specimen was exposed to the laboratory atmosphere for a few hours to induce evaporation, then wrapped up in a plastic cover and left in a humid environment for 24 hours to achieve a homogeneous distribution of the water inside the specimen. It was then weighted for water content determination and a new electrical measurement was taken. Volume changes were determined by measuring with a calliper the current diameter and height. The procedure was repeated a few times to cover the largest possible range of water contents. As long as drying proceeded, samples became progressively more fragile and less conductive, developing small cracks close to the electrodes and making electrical conduction more difficult. No measurements were possible for $w<53 \%$. The experimental relation between measured electrical conductivity and specimen water content is shown in Figure 5.

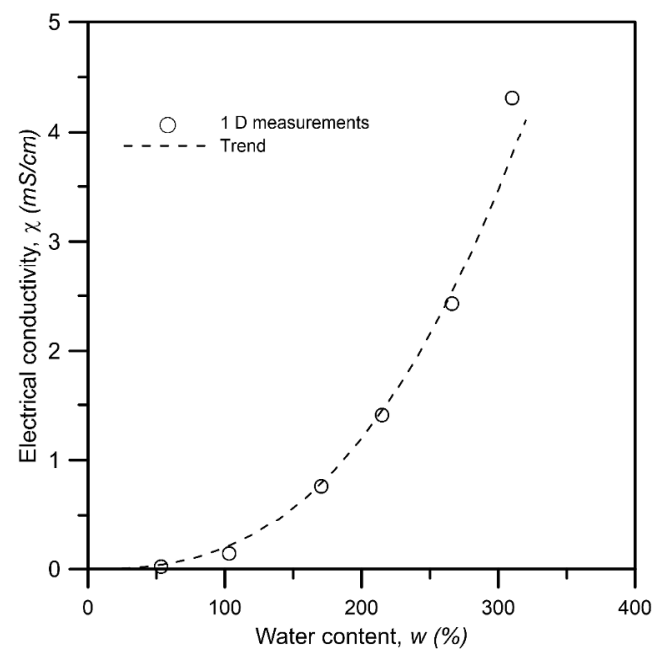

Fig. 5. Dependency of electrical conductivity on the water content. 
The interpolating function depicted in Fig. 5:

$$
\chi=0.28\left(\frac{w}{100}\right)^{2.47}
$$

provides an acceptable fit of the experimental results.

\subsection{Drying of a cylindrical sample}

ERT was employed to monitor the drying process of a specimen having diameter $\mathrm{D}=110 \mathrm{~mm}$ and height $\mathrm{H}=40 \mathrm{~mm}$. The sample was cured for 28 days within a mould similar to the one of Figure 1, then it was placed on a table where it was left to dry to the laboratory environment (temperature $\mathrm{T}=21 \pm 0.5^{\circ} \mathrm{C}$, approximate relative humidity $38 \%$ ). Electrical measurements, and related ERT reconstructions, performed at mid height of the specimen, were repeated along time. An identical sample was placed over a high precision balance. Mass measurements were taken at the same time of the electrical ones, to measure the actual water content at the time of the electrical reconstructions. On basis of mass measurements, the initial water content of this sample was determined to be $\mathrm{w}_{0}=292 \%$, and the corresponding average electrical conductivity $\chi=5.80 \mathrm{mS} / \mathrm{cm}$. The initial value of $\chi$ obtained for this sample is higher than the one used for preliminary characterization, possibly because of different conductivity of the pore fluid or differences in the measurements system. Consistently with both scenarios, it was then assumed that the effects of changes in the water content play the same role in both the ERT reconstructions and in the one dimensional measurements, and equation (2) was corrected as follows:

$$
\chi=0.40\left(\frac{w}{100}\right)^{2.47}
$$

Figure 6 shows the reconstructed maps of electrical conductivity at different times. Maps of water content distribution, estimated by combining the reconstructed electrical conductivity with eq. (3), are shown in Figure 7.

A progressive loss of water occurring quite homogeneously during the first 120 hours can be appreciated. On the contrary, the maps of water content obtained for $\mathrm{t}=144$ hours and $\mathrm{t}=168$ hours, in Fig. $7 \mathrm{~d}$ and Fig. 7 e, are clearly non homogeneous, with water content in the same specimen ranging approximately between $50 \%$ and $160 \%$. Such non homogeneous distribution of water content that were obtained seem mostly due to reconstruction artefacts. In fact, in Figure $7 \mathrm{~d}$ the lowest reconstructed water content is located in the bottom-left part of the specimen, while it is in the upper-right part of the specimen in Figure 7e. This type of changes in the moisture distribution clearly is inconsistent with the boundary conditions that were applied and the hydraulic processes taking place.
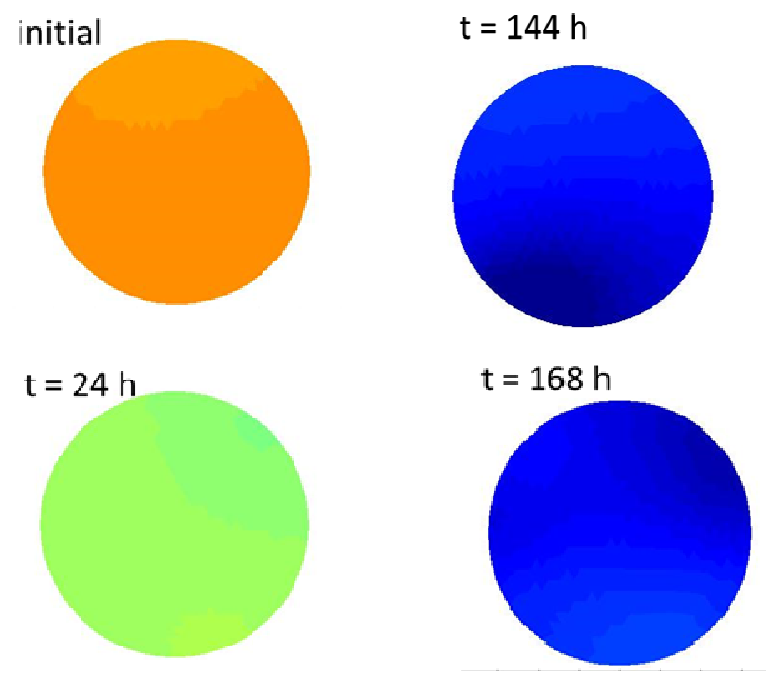

$\mathrm{t}=72 \mathrm{~h}$

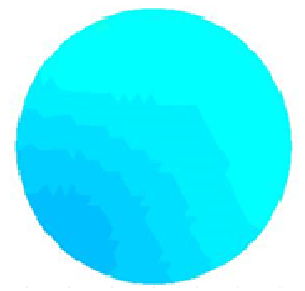

Electrical conductivity $\chi(\mathrm{mS} / \mathrm{cm})$

$\begin{array}{lllllllll}0 & 1 & 2 & 3 & 4 & 5 & 6 & 7 & 8\end{array}$

Fig. 6. Evolution with time of the reconstructed electrical conductivity during drying induced by exposure to laboratory air.
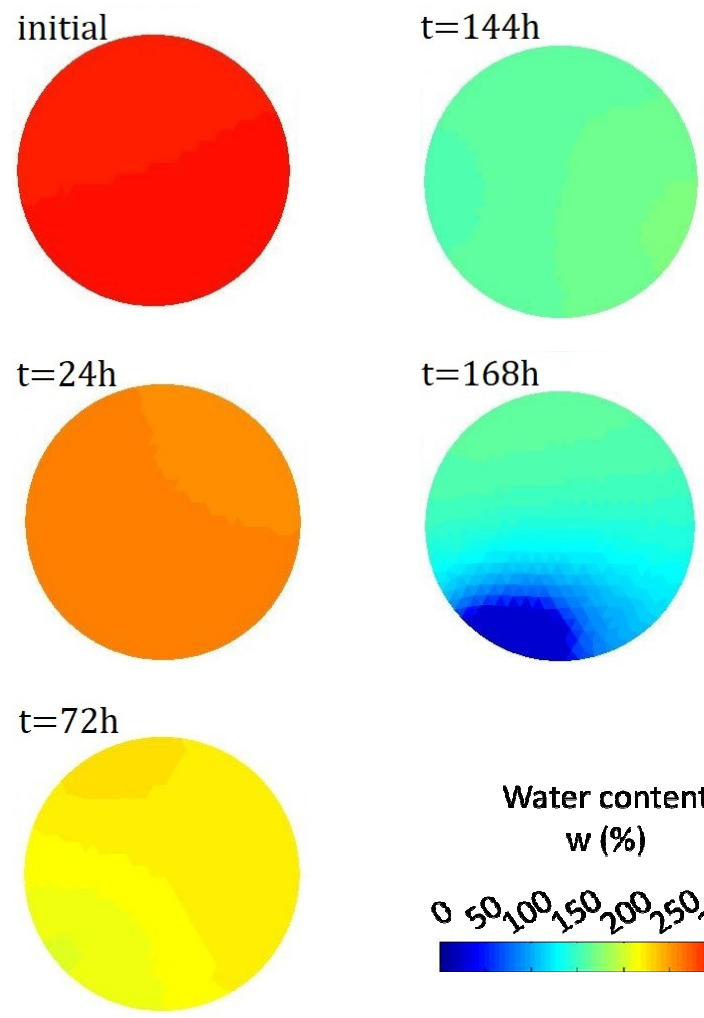

Water content $w(\%)$

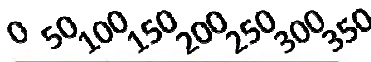

Fig. 7. Distribution of the water content within the sample as predicted by combining eq. 3 with the reconstructed electrical conductivity. 
Interestingly, the average value of the reconstructed water content still compares rather well with the measured one, even when the spatial distribution is not reliable (Figure 8). At larger times (200 h) a discrepancy is found also as far as average values are concerned this discrepancy was found to be related to development of cracks, commented in the last section.

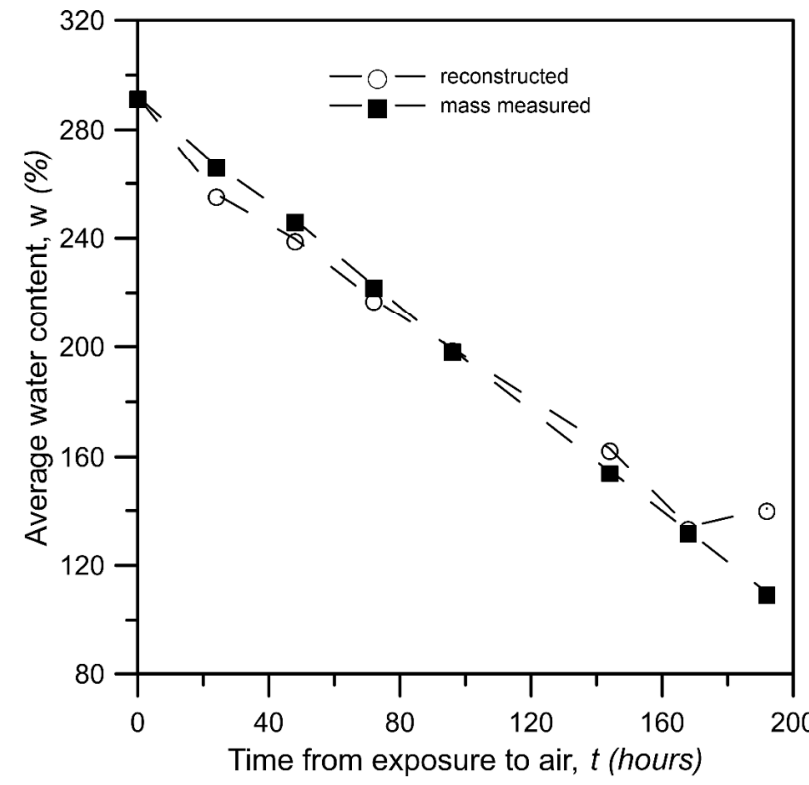

Fig. 8. Evolution with time of the average water content of the sample exposed to laboratory air.

By comparing the maps of reconstructed electrical conductivity (Figs. 6d and 6e) with those of water content (Figs. 7d and 7e), it can be appreciated that the dispersion of the values of the water content is much larger than the one of the electrical conductivity. This is related to the fact that small variations of electrical conductivity in its low range imply large variations of water content (Figure 3).

\subsection{Wetting of a cylindrical sample}

The sample remained exposed to the laboratory environment for another week after the last measurement. It was then placed in a small container that had a $10 \mathrm{~mm}$ height of water at its bottom, to allow for wetting through capillary rise. The maps of reconstructed electrical conductivity and water content in Figure 9 refer to measurements taken 60 hours since the beginning of wetting.

The actual average water content was measured by drying the sample in the oven at $105{ }^{\circ} \mathrm{C}$ for 24 hours, and it was determined to be $\mathrm{w}=265 \%$, while the average reconstructed water content determined through eq. (3) was $\mathrm{w}=271 \%$. Although the moisture content increased significantly upon wetting, the final water content was clearly smaller than the one at the end of curing $\left(\mathrm{w}_{0}=292 \%\right)$. An irreversible decrease of water content, associated to plastic strains, was also found to occur along with drying-wetting cycles in a parallel study [8].

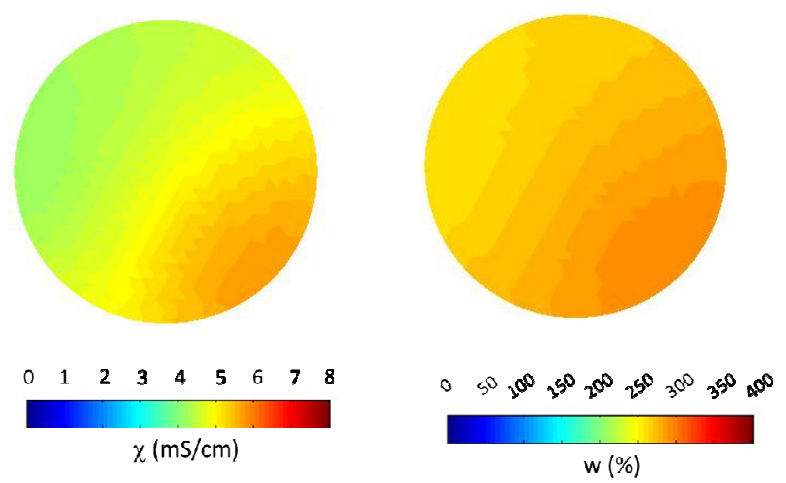

Fig. 9. Maps of reconstructed electrical conductivity and water content obtained 60 hours after wetting induced by contact with $10 \mathrm{~mm}$ of water.

\subsection{Cracking}

No desiccation cracks were visualised on the top of the samples. However, a small vertical crack was observed to develop after six days from the beginning of drying on the side of the sample used for water content determinations (Figure 10). The detected crack might affect to some extent the hydraulic conductivity of the sample, and further experimental efforts are required to evaluate its impact on the hydraulic properties.

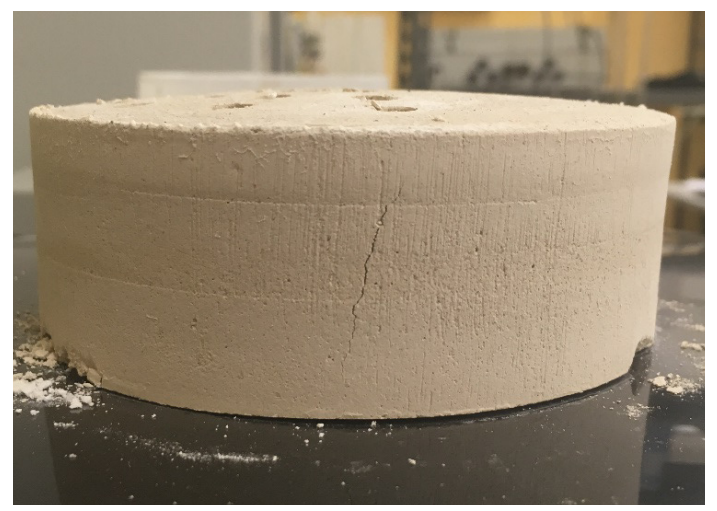

Fig. 10. Small crack developing on the side of the $110 \mathrm{~mm}$ sample [14].

\section{Concluding remarks}

As an attempt to contribute to the study of the effects of drying and wetting on the performance of slurry walls for the containment of contaminants, cement bentonite samples were cured under water in the laboratory for 28 days and then dried through exposure to the laboratory atmosphere. Afterwards, the samples were wetted by placing their bottom in contact with a thin layer of water. Both these processes were monitored through electrical conductivity measurements, interpreted through the ERT technique. The evolution of the water contents was both measured on mass basis and estimated relying on a phenomenological relationship based on the electrical conductivity. The estimated values were very consistent with the measured ones for water contents approximately higher than $\mathrm{w}=100 \%$, which according to the data 
presented it [8] corresponds to matric suction values $s \geq$ $7 \mathrm{MPa}$. At lower water contents, electrical measurements became more difficult, very likely because of poor contact between the electrodes and the sample. The wetting process occurred relatively fast, also in this case a very good correspondence was obtained between the ERT and the mass measurements of water content.

A small crack developed during the drying process. Although limited, cracking might have some impact on the hydraulic performance of the barrier, and further studies are required to characterize it properly. Besides that, ongoing research is focussing on the evaporation processes occurring on cement bentonite columns, and on the effects of drying on the strength of such materials.

\section{References}

1. S.A. Jefferis. In Proc., 10th Int. Conf., International Society for Soil Mechanics and Foundation Engineering, Vol. 1, 425-440 (1981)

2. S. M. Opdyke, J. C. Evans. J. of Geotechnical and Geoenvironmental Engineering, ASCE, 131(6), 673681 (2005)

3. J. M. R. Carreto, L. M. M. S. Caldeira, E. J. L. Maranha das Neves. J. Mater. Civ. Eng., ASCE, 04015093, DOI: 10.1061/(ASCE)MT.19435533.0001365 (2016)

4. K. Soga, K. Joshi, J.C. Evans. In Coupled Phenomena in Environmental Geotechnics. Proc. Int. Symp., ISSMGE TC 315, M. Manassero et al. eds, Taylor and Francis, 149-165, (2013).

5. K. Joshi, Ph.D. Thesis. Dissertation submitted for the degree of Doctor of Philosophy, University of Cambridge, (2009)

6. S.A. Jefferis. In: Johnsen LF, Bruce DA, Byle MJ (eds) Proceedings of the fourth international conference on grouting and deep mixing, New Orleans, 1-24. ISBN: 9780784412350, (2012)

7. A.C.D. Royal, A.W. Opukumo, C. S. Qadr, L. M. Perkins, M. A. Walenna. Geotech Geol Eng, 36, 835 - 853 . DOI: 10.1007/s10706-017-0359-9, (2018)

8. P. Trischitta, R. Cosentini, G. Della Vecchia, G. Sanetti, G. Musso. In E-UNSAT2020 - Unsaturated Horizons $-4^{\text {th }}$ European Conf. on Unsat. Soils -24 - 26 June 2020 Lisboa, Portugal, (2020)

9. A. Borsic, C. Comina, S. Foti, R. Lancellotta, G. Musso, G. Géotechnique 55, 7, 539-547 DOI: 10.1680/geot.2005.55.7.539, (2005)

10. C. Comina, R.M. Cosentini, G. Della Vecchia, S. Foti, G. Musso. Acta Geotechnica 6, (4), 195-203, DOI: 10.1007./s11440-011-0146-3, (2011)

11. R. M. Cosentini, G. Della Vecchia, S. Foti, G. Musso. Géotechnique 62, 7, 583-594 DOI:10.1680/geot.10.P.066 (2012)

12. D. Smyl, M. Hallaji, A. Seppänen, M. Pour-Ghaz. Int. J. of Heat and Mass Transfer, 103, 1348-1358, (2016)
13. D. Smyl, R. Rashetnia, A. Seppänen, M. Pour-Ghaz. Cement and Concrete Research, 91, 61-72, (2017)

14. A. Zibisco, MSc. Thesis, Politecnico di Torino (2019) 